\title{
Die Notariate in der Volksrepublik China
}

\author{
von Harald Richter
}

\section{Vorbemerkung}

Am 13. April 1982 verkündete der Staatsrat der Volksrepublik China eine "Vorläufige Notariatsordnung für die Volksrepublik China ". ${ }^{1}$ Damit wurde, wie ein Kommentar der "Volkszeitung" hervorhebt, ${ }^{2}$ zum erstenmal seit 1949, also seit Bestehen der Volksrepublik China, eine gesetzliche Grundlage für den Ausbau des Notariatswesens geschaffen. Der vollständige Text der neuen Notariatsordnung wird hiermit in deutscher Ubersetzung vorgelegt. Für ein genaues Verständnis der einzelnen Bestimmungen wäre ein ausführlicher Kommentar notwendig - dazu aber fehlen vorerst für die meisten Bereiche verläßliche Einzelinformationen aus der Praxis. In den letzten Jahren ist jedoch über Aufbau und Aufgaben der öffentlichen Notariate häufiger in der chinesischen Presse und in der juristischen Fachliteratur geschrieben worden. Da dieses Material zumindest geeignet ist, einen ersten Einblick in dieses Gebiet der Rechtspflege in China zu vermitteln, wurde es zu einem begleitenden Kommentar verarbeitet, der die Bestimmungen der neuen Notariatsordnung ergänzen soll. ${ }^{3}$

\section{Notariate im "sozialistischen Rechtssystem" - Neubeginn nach dem Sturz der "Vie- rerbande"}

Wiederherstellung und Ausbau eines "sozialistischen Rechtssystems" (shehuizhuyi fazhi) gehören zu den programmatischen Forderungen, die auf dem entscheidenden 3. Plenum des XI. Zentralkomitees der chinesischen KP im Dezember 1978 aufgestellt wur$\operatorname{den}^{4}$

1 "Zhonghua Renmin Gongheguo gongzheng zanxing tiaoli", vgl. Renmin Ribao (im Folgenden abgekürzt RM) vom 25. 4. 1982, Guangming Ribao (im Folgenden abgekürzt GM) vom 25. 4. 1982 und Xinhua News Bulletin (Englisch) vom 24. 4. 1982 ("Notary Public Regulations Issued ") jeweils mit einem Uberblick über die wichtigsten Regelungen. Der vollständige chinesische Text wurde erst am 29.6. 1982 in Renmin Ribao veröffentlicht, desgleichen im Guowuyuan Gongbao (Bulletin des Staatsrates) Nr. 384 vom 10.7. 1982, S. 451-454.

2 Vgl. RM 29. 6. 1982 Kurzkommentar "Die Arbeit der öffentlichen Notariate gewissenhaft durchführen".

3 Der Verfasser dieses Artikels besuchte am 27. 11. 1981 das Notariat der Stadt Peking. Die Informationen aus dem Gespräch mit einem stellvertretenden Leiter des Notariats sind ebenfalls in dem vorliegenden Artikel verarbeitet. - Für Hinweise, Verbesserungsvorschläge sowie die Durchsicht des Manuskriptes dieses Artikels dankt der Verfasser Wolf gang Keßler, Hamburg.

4 RM 24. 12. 1978. 
Die 'Kulturrevolution und ihre Folgezeit hatten zehn Jahre des Chaos, der politischen Willkür, der Rechtsunsicherheit und Rechtlosigkeit, der Abkehr von einem in China seit 1949 ohnehin nur bruchstückhaft entwickelten kodifizierten Recht mit sich gebracht. Ein gründlicher Neubeginn war geboten, wollte man das Vertrauen der Bevölkerung in Staat und Verwaltung wenigstens schrittweise wiederherstellen und dem Volk allmählich wieder ein Gefühl der Rechtssicherheit vermitteln.

$\mathrm{Zu}$ den Organen der Rechtspflege, die im Rahmen des "Aufbaus des sozialistischen Rechtssystems" ihre durch die 'Kulturrevolution' unterbrochene Arbeit wieder aufnehmen konnten, gehören auch die Notariate (gongzhengchu). Ende 1979 forderte die "Volkszeitung" in einem Artikel, ${ }^{5}$ die Einrichtung von Notariaten zu beschleunigen. Dies sei wegen der starken Zunahme wirtschaftlicher Aktivitäten im Inland - infolge einer weniger dogmatischen Wirtschaftspolitik - und wegen der Intensivierung der internationalen Kontakte Chinas notwendig. Ab 1980 berichtete die Presse häufiger über Probleme der Notariate und begann, die Bevölkerung über Funktion, konkrete Aufgaben und Aufbau der Notariate zu informieren, um verbreitete Mißverständnisse auszuräumen und die Bedeutung der Tätigkeit der Notariate den Massen verständlich zu machen. Eine Reihe von Konferenzen wurde abgehalten, um eine Bestandsaufnahme vorzunehmen und sich über die zukünftige Entwicklung Gedanken zu machen. Auf einer "Nationalen Arbeitskonferenz der Justiz-Verwaltung" vom 30. 7.-9. 8. 1980 in Peking wurde als einer der wichtigsten Aufgabenbereiche der weitere Ausbau der Notariate genannt. ${ }^{6}$ Etwa einen Monat später, vom 19.-25. 9.1980 fand in Peking eine nationale Arbeitskonferenz ausschließlich über die Notariate statt. Diskutiert wurden die Erfahrungen bei der bisherigen Arbeit, die Aufgaben der Notariate in der neuen Phase wirtschaftlicher und gesellschaftlicher Umstrukturierungen, Fragen der Organisation, des fachlichen Aufgabenrahmens, der Kader-Ausbildung und der Aufklärungsarbeit in der Bevölkerung. Auch der Entwurf einer "Notariatsordnung für die Volksrepublik China" (Zhonghua Renmin Gongheguo Gongzheng Tiaoli) kam zur Sprache. ${ }^{7}$ Ende 1980 berichtete das Justizministerium (sifabu) bereits von "großen Fortschritten" beim Ausbau der Notariate und der Ausweitung des Personalbestandes und nannte erstmals Zahlen: danach bestanden über 350 Notariatsbehörden in 20 Provinzen, mit einem Personal von insgesamt über 1000 Personen. ${ }^{8}$ Aufbau und Ausbau des Notariatswesens sind noch

5 Yang Rongxin, "Jiasu tuixing gongzheng zhidu" (Das System der öf fentlichen Notariate beschleunigt ausbauen), RM 18. 12. 1979.

6 Zhongguo Baike Nianjian (Enzyklopädisches China-Jahrbuch) 1981, S. 189. Der vollständige Text des Berichtes Justizministeriums über diese Konferenz ist abgedruckt im Guowuyuan Gongbao, Nr. 347, vom 31. 12. 1980, S. 639-645. Es werden zehn "allmählich zu vollendende Aufgaben " genannt, darunter unter Nr. 6 die Organisation der Arbeit der Notariate und Rechtsanwälte. Die Konferenz beschäftigte sich auch mit Themen wie dem Nichtvorhandensein von Dienst-Uniformen für Notare (S. 643) sowie den Amtstiteln der Notare (S. 644).

7 Hu Heng, "Zuohao gongzheng gongzuo weihu gongmin hefa quanyi" (Die notarielle Arbeit gut durchführen um die Rechte und Interessen der Bürger zu schützen), GM 28. 9. 1980; s. auch Enzyklopädisches ChinaJahrbuch 1981, S. 190 und RM 3. 10. 1980.

8 RM 5. 11. 1980. 
nicht abgeschlossen, jedoch ist die auf der Konferenz vom September 1980 diskutierte Notariatsordnung inzwischen verkündet worden und regelt, wenn auch nicht im Detail, den Aufbau, die Aufgaben und Zuständigkeiten der Notariate. Die Bezeichnung "vorläufige Notariatsordnung " läßt darauf schließen, daß nach einer gewissen Zeit der Erprobung in der Praxis eine Úberarbeitung möglich ist.

\section{Zur Geschichte der Notariate in der Volksrepublik China}

Um die Notwendigkeit der Einrichtung von Notariaten herauszustreichen bemüht man sich, diese gegenüber früher gebräuchlichen Formen privater Beurkundungen abzugrenzen: China kannte in früherer Zeit die privaten Beurkundungen durch Zeugen oder Vermittler (zhongren), z. B. bei Landverkäufen, Hausverkäufen, Erbschaften, Teilungen von Familienvermögen, Geldverleih usw. Anläßlich des jeweiligen Rechtsgeschäftes wurden lokale Würdenträger zum Bankett eingeladen und setzten als Zeugen ihre Unterschrift unter schriftlich ausgefertigte Urkunden, in denen festgestellt wurde, daß eine jeweilige Aktion tatsächlich stattgefunden hatte. Mehr als dies konnten jene privaten Beurkundungen nicht feststellen, also z. B. nicht, ob die Angelegenheit fair und korrekt und den Gesetzen entsprechend durchgeführt wurde. Da so die rechtlichen Interessen der Beteiligten nur ungenügend geschützt wurden, konnten Meinungsverschiedenheiten nicht ausgeschlossen werden. ${ }^{9}$

Die Volksrepublik China betont den öffentlichen Charakter der Notariate als staatliche Organe der sozialistischen Rechtspflege. Die Notariate sollen dem Allgemeininteresse aller Bürger dienen. Die Ursprünge des Notariatswesens in der Volksrepublik China gehen zurück auf rudimentäre Ansätze in den sogenannten "befreiten Gebieten", also schon vor der offiziellen Proklamierung der Volksrepublik. Die Entwicklung des Notariatswesens nach 1949 blieb jedoch stets abhängig von der politischen Lage und war somit starken Schwankungen unterworfen. Der Aufbau ist, nach eigenem Eingeständnis, aus "einer Reihe von Gründen " bisher nur unvollständig gelungen. ${ }^{10}$

Bereits 1948 hatte man in der Stadt Harbin nach der Einnahme durch kommunistische Truppen ${ }^{11}$ begonnen, Notariate einzurichten, deren Arbeitsbereich jedoch relativ klein war. ${ }^{12}$ Erst nach Oktober 1949 begann allmählich der Aufbau von Notariaten in den großen und mittleren Städten sowie in einer Reihe von Landkreisen. Entsprechend den Bestimmungen in Artikel 12 und 20 des "Vorläufigen Organisations-Statuts für die Volksgerichte der Volksrepublik China" vom 3. 9. 1951 wurden diese Notariatsbehörden den Volksgerichten beigeordnet.

9 RM 18. 12. 1979.

10 RM 18. 12. 1979.

11 RM 18. 12. 1979, gibt als Jahr 1948 an, GM 28. 3. 1980 nennt als Jahr 1946. Letzteres ist wohl korrekt, denn das of fizielle Datum der 'Befreiung' Harbins wird mit dem 28. 4. 1946 angegeben, vgl. Shishi Ziliao Shouce, Beijing 1981, S. 180.

12 RM 18. 12. 1979. 
Im Verlauf der Bewegung zur "sozialistischen Umgestaltung der kapitalistischen Industrie und des Handels" (1953-1956) nahm die Zahl der zwischen Staat und damals noch privaten Betrieben geschlossenen Wirtschaftsverträge (jingji hetong) stark zu. Diese Verträge wurden jedoch oft ("von den Kapitalisten") nicht eingehalten, und man schätzte, daß die Fälle von Vertragsverletzungen einen Prozentsatz zwischen 20-25\% erreichten. ${ }^{13}$ Um hier Abhilfe zu schaffen, sollten durch öffentliche Beurkundung solche Verträge wirksamer gemacht werden. Zu diesem Zweck richtete man - zuerst in der Stadt Nanchang (Provinz Jiangxi) ${ }^{14}$ - später auch in anderen großen und mittleren Städten Notariate ein, die sich speziell mit diesen Wirtschaftsverträgen befaßten. Die "sozialistische Umgestaltung" erforderte bald eine Ausweitung der notariellen Tätigkeiten auf andere Bereiche, z. B. Beglaubigung von Testamenten, Verschollenheitserklärungen, Verwaltung von Vermögen, Sicherung von Beweismitteln u. a. ${ }^{15}$

Im Juli 1956 bestimmte der Staatsrat, daß in Städten mit über 300000 Einwohnern sowie in industriell entwickelten Städten und Landkreisen, in denen in größerer Zahl Verwandte von Auslandschinesen lebten, Notariate einzurichten seien. In anderen Städten und Kreisen sollten bei den Volksgerichten Notarbüros eingerichtet werden oder die notariellen Aufgaben von den Richtern der Volksgerichte mit wahrgenommen werden. ${ }^{16}$ Am 18. 9. 1956 erließen Finanz- und Justizministerium gemeinsam eine Weisung bezüglich der "Bestimmungen über das Verfahren zur Festsetzung von Notargebühren und über das Verfahren zur Subventionierung der Ausgaben der Notariatsbehörden “. ${ }^{17}$ Auf dem VIII. Parteitag der Kommunistischen Partei Chinas (1956) lobte Dong Biwu, einer der Parteigründer und damals Präsident des Obersten Volksgerichts (1954-1959) das System der Notariate und forderte deren raschen Ausbau. ${ }^{18}$ Die erste Blütezeit des No-

13 Wu Kexian, "Tantan woguo de gongzheng gongzuo" (Uber die notarielle Arbeit in unserem Lande), GM 28. 3. 1980. Nach Einführung der Beurkundung, so heißt es in dem Artikel, sei der Prozentsatz der Vertragsverletzungen auf $1-5 \%$ gesunken.

14 GM 28. 3. 1980.

15 GM 28. 3. 1980 zählt für diese Zeit bereits "über zwanzig verschiedene Arten“ der notariellen Tätigkeit.

16 GM 28. 3. 1980.

17 "Guanyu zhiding gongzhengfei guanli banf a he gongzheng jiguan kaizhi buzhu banfa de guiding de tongzhi“ vom 18. 9. 1956:

"Entsprechend dem vom Staatsrat mit Schreiben vom 10. 7. 1956 genehmigten Bericht des Justizministeriums über die Arbeit der Notariate wird festgelegt, daß über das konkrete Verwaltungsverfahren bezüglich der Notargebühren und das Verfahren für die Subventionierung der Ausgaben der Notariatsorgane in Konsultation zwischen dem Justiz- und dem Finanzministerium entschieden wird،.

Um den konkreten Umständen der jeweiligen Orte Rechnung zu tragen und um den jeweiligen Justiz-Ämtern (sifating/sifaju) die geplante Einrichtung von Notariaten, deren personelle Ausstattung sowie die Abstimmung der Pläne bezüglich der Sätze von Notargebühren zu erleichtern und deren reibungslose Durchführung zu gewährleisten, wird auf grund der Prüf ung durch die beiden Ministerien beschlossen, daß die Zentral(regierung) keine einheitliche Regelung trifft. Die Justiz-Ämter sowie die Finanz-Ämter (caizhengting/caizhengju) aller Provinzen, (Autonomen) Gebiete und Regierungsunmittelbaren Städte werden hiermit bevollmächtigt, gemeinsam Verwaltungsverfahren für die Notargebühren und Verfahren für die Subventionierung der Ausgaben der Notariatsorgane festzulegen, die den Volkskomitees der entsprechenden Ebene zur Billigung und Durchführung vorgelegt werden und die (unseren) beiden Ministerien jeweils zur Kenntnis gebracht werden". In: Zhonghua Renmin Gongheguo Fagui Huibian, Band 4, Juli-Dezember 1956, Seite 242.

18 GM 28. 3. 1980. 
tariatswesens fiel in das Jahr 1957: in 50 Städten im gesamten Land hatte man eigenständige Notariate eingerichtet, in 501 Städten und Landkreisen bestanden Notarbüros bei den örtlichen Volksgerichten, und 552 andere Kreis-Volksgerichte erfüllten gleichzeitig notarielle Aufgaben. ${ }^{19}$ Aber noch im Jahr 1957 begann, ausgelöst durch die "AntiRechts-Kampagne" (fan youpai douzheng), eine Phase der Geringschätzung des Rechtes. Der Ausbau des Notariatswesens kam infolge der politischen Ereignisse zunehmend ins Stocken. 1958, in der Zeit des "Großen Sprungs nach Vorn" und der Errichtung der "Volkskommunen", sah sich das System der Notariate mit "Störungen" und Mißachtung konfrontiert, der fachliche Arbeitsrahmen wurde eingeschränkt und später die Arbeit im Innenverkehr (s. unten) ganz eingestellt. ${ }^{20}$ Nach 1959 behielten nur wenige Großstädte wie Peking, Shanghai und Kanton ihre Notariate, die ihre Arbeit auch nur weiterführten, um den nach internationaler Praxis notwendigen Aufgaben im Außenverkehr gerecht zu werden. ${ }^{21}$ Die Schließung der Notariate steht sicher auch im Zusammenhang mit der vom II. Nationalen Volkskongreß am 28. 4. 1959 beschlossenen Auflösung des Justizministeriums, ${ }^{22}$ das als oberste Justiz-Verwaltungsbehörde auch Aufsichts-Organ der Notariate war.

In den politischen Wirren der "Kulturrevolution" und deren Folgezeit kam die notarielle Arbeit fast völlig zum Erliegen. Es wird berichtet, daß selbst Auslandschinesen, die für ihre Arbeit oder für ihr Studium im Ausland notariell beglaubigte Urkunden aus China benötigten, keine Hilfe mehr von ihren Verwandten erhielten. Diese wagten aus Furcht vor Verwicklungen oder Verdächtigungen nicht, die wenigen, noch offenen Notariate zu kontaktieren.

Erst nach dem Sturz der "Viererbande" konnte sich die Arbeit der Notariate wieder entfalten. Im September 1979 wurde nach zwanzigjähriger Unterbrechung das Justizministerium wieder errichtet. ${ }^{23}$ Die Notariate wurden zunächst überwiegend im Außenverkehr (s. unten) aktiv. Ein Beispiel aus Süd-China bezeugt dies: vom Notariat der Stadt Foshan, in der Nähe von Kanton gelegen, wird berichtet, daß dort von April bis Juni 1980 etwa 12000 Anträge auf Beurkundung bearbeitet worden seien. ${ }^{24}$ Hier spiegelt sich natürlich die hohe Zahl der Auslandschinesen bzw. der in Hongkong lebenden Chinesen wider, die aus dieser Gegend stammen und noch verwandtschaftliche Beziehungen dort haben.

19 Si Zhengwen und Hu Heng, "Quanguo gongzheng gongzuo zai jiji kaizhan zhong" (Die Arbeit der Notariate im ganzen Land entfaltet sich aktiv), GM 15. 8. 1980.

20 GM 28. 3. 1980 und 15. 8. 1980.

21 GM 15. 8. 1980.

22 Vgl. Lan Quanpu, Sanshi Nian Lai Woguo Fagui Yange de Gaikuang (Dreißig Jahre Entwicklung des Rechtes in unserem Lande), Peking: Qunzhong Chubanshe, 1980, S. 156.

23 RM 14. 9. 1979 und 19. 9. 1979.

24 GM 15. 8. 1980. 


\section{Der Aufbau der Notariate}

Bis zur Verabschiedung der "Vorläufigen Notariatsordnung" von 1982 gab es kein Gesetz, das ausschließlich die Organisation der Notariate regelte. Das "Vorläufige Organisationsstatut für die Volksgerichte der Volksrepublik China «"25 vom 3. 9.1951 erwähnte jedoch die Notariate und bestimmte in den Artikeln 12 und 20, daß die Wahrnehmurig notarieller Aufgaben ein Teil der Arbeit der unteren und mittleren Volksgerichte war. Im Gefolge dieser Bestimmungen wurden bei den Volksgerichten weiniger Kreise und Städte ${ }^{26}$ Notariate eingerichtet. Jene Zeit wird als "Anfangsstadium des Notariatswesens ${ }^{27}$ bezeichnet. Der Aufbau der Volksgerichte ${ }^{28}$ ist inzwischen in einem am 1. Juli 1979 vom Nationalen Volkskongreß verabschiedeten neuen Gerichtsverfassungsgeset ${ }^{29}$ geregelt worden, in dem sich keine direkte Erwähnung der Notariate mehr findet. In Artikel 22 Abs. 3 heißt es lediglich, daß die Volksgerichte der unteren Ebene neben der Rechtsprechung auch "im Rahmen der von den höheren Justizverwaltungsbehörden übertragenen Aufgaben und Befugnisse Arbeiten der Justizverwaltung durchführen". Die Notariate sind nach heutigem chinesischen Verständnis staatliche Organe (dies wird auch in Artikel 3 der neuen Notariatsordnung ausdrücklich festgestellt) unter Leitung bzw. Aufsicht der Justizverwaltungsbehörden (sifa xingzheng bumen), ${ }^{30}$ nicht jedoch Unterabteilungen der Volksgerichte. Lediglich räumlich sind die Notariate meist bei den jeweiligen Volksgerichten untergebracht.

Aus bisherigen Presseveröffentlichungen ${ }^{31}$ läßt sich über die Organisation der Notariate in der Vergangenheit etwa folgendes sagen: In allen großen und mittleren Städten, in denen es Justizverwaltungsbehörden gab, sollten auch Notariate (gongzhengchu) eingerichtet werden, die ausschließlich notarielle Aufgaben wahrnahmen. In Kreisen und Städten der untersten Ebene sollten entsprechend der Bevölkerungszahl sowie entspre-

25 "Zhonghua Renmin Gongheguo Renmin Fayuan Zanxing Zuzhi Tiaoli“, Text in: Zhongyang Renmin Zhengfu Faling Huibian (1951) (Sammlung von Gesetzen und Verordnungen der zentralen Volksregierung), S. 79.

26 GM 28. 3. 1980.

27 GM 28. 3. 1980.

28 Volksgerichte bestehen auf vier Ebenen: (1) Untere Ebene: Volksgerichte der (Land)Kreise, kreisfreien Städte und (in Großstädten) der einzelnen Stadtbezirke; (2) Mittlere Ebene: Volksgerichte der Regierungsbezirke bzw. der Städte auf dieser Verwaltungsebene; (3) Höhere Ebene: Volksgerichte der Provinzen (jeweils eines für jede Provinz); (4) Oberster Volksgerichtshof - vgl. §§ 2, 18, 23, 26, 30 GVG-VRCh (1979), Fn. (29).

29 Zhonghua Renmin Gongheguo Renmin Fayuan Zuzhifa, Text in: Gong' an Fagui Huibian, Peking: Qunzhong Chubanshe, 1981, S. 449; vgl. Kommentar dazu Sun Yingjie/Feng Caijin (Hrsg.), Zhonghua Renmin Gongheguo Renmin Fayuan Zuzhif a Jianghua, Peking: Qunzhong Chubanshe, 1980.

30 Die Justizverwaltung in China wird von besonderen Justizverwaltungsbehörden durchgeführt, an deren Spitze das 1979 wieder eingerichtete Justizministerium (sifabu) steht. Die Behörden sind den jeweiligen örtlichen Volksregierungen unterstellt, sie führen die Bezeichnung "sif aju" oder "sifating" (auf Provinzebene) und "sifaju" oder "sifachu" (in Städten bzw. Regierungsbezirken). Ihre Auf gaben sind u. a. die Einrichtung von Volksgerichten (organisatorisch), Planung und Änderung bezüglich des Amtsbezirks der Gerichte, Finanzverwaltung, Personalangelegenheiten, Ausbildung des Gerichtspersonals, Ausgabe von Gesetzes- und Verordnungs-Sammlungen, Koordinierung rechtswissenschaftlicher Forschung, Verwaltung der Hochschulen für Politik und Recht, Uberwachung der Rechtsanwälte und der Notariatsbehörden. - vgl. Falü Changshi Shouce, Peking, 1979, S. 12; Faxue Cidian, Shanghai, 1980, S. 200; Lan Quanpu a.a.O., S. 155-156.

31 Besonders Tang Bai, "Shenmo shi gongzheng" (Was ist öf fentliche Beurkundung), GM 5. 5. 1981. 
chend den örtlichen Erfordernissen und Möglichkeiten entweder besondere Notariate eingerichtet, oder bei den Volksgerichten der unteren Ebene Notarbüros (gongzhengshi) eingerichtet bzw. besondere Beamte mit der Wahrnehmung notarieller Aufgaben (gongzheng yewu) beauftragt werden. ${ }^{32}$ Für das Jahr 1957 gibt es genaue Angaben über die Zahl der in ganz China bestehenden Notariate (s. oben), aufgeschlüsselt jeweils nach der Art des Notariats. Angaben über den Standort stehen jedoch nicht zur Verfügung, auch ist fraglich, ob an allen Orten mit den notwendigen Voraussetzungen auch tatsächlich Notariate eingerichtet worden waren. Heute ist die Zahl der vorhandenen Notariate in etwa wieder bekannt, ${ }^{33}$ ohne Aufschlüsselung nach Art und Größe. Als räumlicher Zuständigkeitsbereich wird jeweils Stadt, Kreis oder Regierungsbezirk genannt. ${ }^{34}$ Auch Stadtbezirke in Großstädten verfügen über Notariate.

Das Fachpersonal der Notariate bestand bisher aus Notaren (gongzhengyuan), assistierenden Notaren (zhuli gongzhengyuan) sowie den Leitern der Notariate (zhuren gongzhengyuan). ${ }^{35}$ Sie sind Funktionäre des Staates (guojia ganbu), ihre Qualifikation sollte derjenigen der Richter entsprechen. Bezüglich Bestallung und Entlassung galten die Richtlinien für Verwaltungsfunktionäre. Die Notare erhielten ein festes Gehalt, die Einnahmen der Notariate gingen an die Staatskasse. ${ }^{36}$

Die neue Notariatsordnung schreibt im wesentlichen die bisher bekannten bzw. in der Praxis herausgebildeten Verhältnisse fest und regelt im Abschnitt III den Aufbau und die personelle Ausstattung der Notariate. Sie enthält jedoch auch genauere Bestimmungen (Art. 8 und 9) darüber, welcher Personenkreis unter welchen Bedingungen zum Notar bzw. assistierenden Notar berufen werden kann.

\section{Die Funktion der Notariate}

Die Notariate sind Organe der sozialistischen Rechtspflege. Ihre grundlegende Aufgabe besteht in der öffentlichen ${ }^{37}$ Beurkundung von Rechtsgeschäften (falü xingwei) und

32 GM 15. 5. 1981 berichtet, daß in Orten, wo noch keine Notariate eingerichtet wurden, Anträge auf notarielle Beurkundung für den Außenverkehr bei den Volksgerichten zu stellen sind.

33 Die seit Mitte 1980 gemachten Zahlenangaben spiegeln recht gut die rasche Zunahme der Zahl der Notariate wider: Im August 1980 werden 154 Notariate mit einem Personalbestand von etwa 1000 Personen genannt (GM 15. 8. 1980), im November 1980 ist bereits von über 350 Notariaten in 20 Provinzen, mit einem Personalbestand von über 1000 Personen die Rede (RM 5. 11. 1980). Anläßlich der Verkündung der Notariatsordnung im April 1982 nennt Renmin Ribao 1596 Notariate mit einem Personalbestand von 3170 Personen; im Juni 1982 spricht der Begleitkommentar zur Veröffentlichung des Textes der Notariatsordnung von "1600 Notariaten in Städten, Kreisen und Regierungsbezirken“ (RM 29. 6. 1982). Damit wären die Zahlen von 1957 bereits überschritten.

34 RM 29. 6. 1982.

35 GM 28. 3. 1980.

36 RM 18. 12. 1979; GM 28. 3. 1980; zur Regelung der Gebührenfrage vgl. Fn. (17). Beim Besuch im Pekinger Notariat im November 1981 wurde dem Verfasser gesagt, die Gebühren seien einheitlich im ganzen Land geregelt.

37 Die chinesischen Autoren betonen immer wieder, daß es sich um öffentliche Beurkundung handelt, im Gegensatz zu den privaten Beurkundungen, die weder die Korrektheit noch die Rechtmäßigkeit eines Rechtsgeschäftes hätten feststellen können. 
rechtlich relevanten Dokumenten oder Tatsachen (jou falü yiyi de wenshu huo shishi), d. h. in der Feststellung, daß Dokumente von rechtlicher Bedeutung inhaltlich den Tatsachen entsprechen und mit dem geltenden Recht in Einklang stehen. Nach chinesischer Auffassung ist die Durchführung notarieller Akte notwendig, um

- das öffentliche Eigentum (gonggong caichan) zu schützen;

- das individuelle Eigentum (geren caichan) der Bürger zu schützen; ${ }^{38}$

- die Rechte und legalen Interessen von staatlichen Organen, Betrieben und Organisationen (tuanti) zu schützen; ${ }^{39}$

- die Rechte und legalen Interessen der Bürger hinsichtlich ihres Personenstandes und Eigentums zu schützen. ${ }^{40}$

Die Arbeit der Notariate hat sowohl eine "erzieherische" als auch eine "präventive" Funktion. ${ }^{41}$ Die Notariate sollen dazu beitragen, die Bürger zur Achtung vor dem Recht zu erziehen, ${ }^{42}$ ihr Rechtsbewußtsein zu stärken und somit für die Festigung der staatlichen Rechtsordnung zu wirken. "Präventiv" (yufang) wirkt die Arbeit insofern, als Streitigkeiten vermieden, die Zahl der Gerichtsprozesse verringert und die Anlässe für Prozesse bereits im Vorhinein ausgeräumt werden können.

Angesichts der Tatsache, daß der Aufbau eines umfassenden Notariatswesens in China bisher unvollständig geblieben ist, die Notariate also noch eine neue Einrichtung sind, ist verständlich, daß man die Bürger über Arbeit und Funktion dieser Behörde aufklären muß, um Mißverständnisse und Fehleinschätzungen zu begegnen. Von der Bevölkerung werden die Notariate offenbar vielfach als eine weitere bürokratische Institution betrachtet, die lediglich der "Erledigung von Formalitäten" (banli shouxu) dient. Es wird sogar berichtet, daß es innerhalb der Justizbehörden die Ansicht gibt, die Arbeit der Notariate sei "nicht wesentlich". In einem Artikel in der juristischen Fachzeitschrift "Faxue Yanjiu ", ${ }^{43}$ der über diese Fehleinschätzungen berichtet, bemüht sich der Autor, deutlich zu machen, daß

- Notariate nicht durch private Beurkundungen ersetzt werden können;

- Notariate spezielle Funktionen haben, die nicht von anderen Behörden übernommen werden können;

- die Arbeit der Notariate ein wichtiges Element bei der Regelung zivilrechtlicher Beziehungen ist;

- die Notare juristisches Fachpersonal sind.

Die Notariate sind allerdings nicht mit Gerichten zu verwechseln: auf die Unterschiede

38 Privates Eigentum bzw. das Erbrecht wird in Artikel 13 der neuen Verfassung von 1982 garantiert, vgl. Beijing Rundschau Nr. 52, 1982, S. 11.

39 Dies sind die juristischen Personen (faren).

40 GM 5. 5. 1981.

41 GM 5. 5. 1981; vgl. den Gesichtspunkt der "vorsorgenden Rechtspflege" im Zusammenhang mit der Arbeit der Notare in Deutschland ( $\$ 24$ Bundesnotarordnung).

42 GM 5. 5. 1981; vgl. auch Artikel 3 der Notariatsordnung, in den diese Auf gabe ausdrücklich hineingeschrieben wurde.

43 Shi Fengyi, "Gongzheng zhidu zai fazhi jianshe zhong de diwei he zuoyong" (Stellung und Funktion des Notariatswesens beim Aufbau des Rechtssystems), Faxue Yanjiu Nr. 2, 1981, S. 41. 
der Funktion und Arbeit beider Institutionen glaubte man, in der Presse speziell aufmerksam machen zu müssen: ${ }^{44}$ Volksgerichte üben das Urteilsrecht (shenpanquan) aus, und die von den Gerichten gefällten Entscheidungen sind bindend, sie können in Form eines Urteils (panjue), eines Beschlusses (caiding) oder einer gerichtlichen Schlichtung (tiaojie) gefaßt werden. Die jeweils darüber ausgestellten Urkunden ${ }^{45}$ haben rechtlich bindende Wirkung.

Beschlüsse und Urteile können die zivilrechtlichen Beziehungen (Rechte und Pflichten) ändern und modifizieren, die Urkunden der Notariate können dies nicht, denn die Notariate haben keine Befugnis, gerichtliche Entscheidungen zu fällen. Notarielle Urkunden haben jedoch rechtliche Beweiskraft (zhengju de xiaoli). Bei Zivilprozessen können solche Urkunden als besondere Beweisurkunde (teding shuzheng) in Fällen entsprechender gesetzlicher Formerfordernisse verwendet werden, sie können direkt als Beweismittel (zhengju) akzeptiert werden, wenn seitens des Gerichtes keine Zweifel bestehen. Die Volksgerichte, die sich nicht allein auf den Beweis-Rahmen der notariellen Urkunden beschränken dürfen, können unterschiedliche Beschlüsse und Urteile bezüglich der von den notariellen Urkunden beurkundeten Inhalte treffen. ${ }^{46}$

In der praktischen Arbeit der Notariate sollen folgende Grundsätze beachtet werden: ${ }^{47}$

(1) Der Grundsatz der Freiwilligkeit der Beurkundung (ziyuan gongzheng): Eine erzwungene notarielle Beurkundung (qiangzhi gongzheng) ist nicht erlaubt; ${ }^{48}$

(2) Der Grundsatz der "Erleichterung für die Massen" (bianli qunzhong): Wenn die beteiligten Parteien unter bestimmten Umständen nicht persönlich erscheinen können, muß das Personal der Notariate diese zu Hause aufsuchen, um seine Aufgaben wahrzunehmen;

(3) Der Grundsatz der "Ausschließung" (huibi): Die Bearbeitung aller notariellen Angelegenheiten von Verwandten des Notariatspersonals muß vermieden werden, um keinen Anlaß zu Interessenkonflikten zu geben; ${ }^{49}$

(4) Der Grundsatz der Verschwiegenheit (baomi): Da die beurkundeten Angelegenheiten meistens den Personenstand und die Eigentumsverhältnisse betreffen sowie Rechte und Pflichten der beteiligten Parteien, dürfen sie nicht veröffentlicht werden. ${ }^{50}$

44 GM 1. 8. 1981, Chen Liushu, "Gongzhengshu yu caiding, panjue“ (Notarielle Urkunden und Beschlüsse, Urteile).

45 Genannt "falü wenshu“, unterschieden wird zwischen "panjueshu" (Urteilen) und "caidingshu" (Beschlüssen).

46 Zur "besonderen Beweisurkunde“ vgl. Tang Dehua, Yang Rongxin, Cheng Yanling, Zhu Xisen, Minshi Susongfa Jiben Zhishi (Grundwissen zum Zivilprozeßrecht), Peking: Falü Chubanshe, 1981, S. 98; zur Beweiswürdigung s. § 55 Abs. 2 ZPO-VRCh (1982); zu Ubersetzungen der ZPO vgl. unten Fn. (127).

47 GM 28. 3. 1980.

48 Ausnahme: Wirtschaftsverträge in den 50er Jahren. Das Prinzip der Freiwilligkeit wird in Artikel 2 der neuen Notariatsordnung implizit angesprochen: "auf Antrag der beteiligten Parteien“. Das neue Gesetz über Wirtschaftsverträge (vgl. Fn. [129]) schreibt übrigens keine notarielle Beurkundung bestimmter Vertragstypen zwingend vor.

49 GM 28. 3. 1980. Dieser Punkt ist etwas unklar ausgedrückt, betrifft aber wohl den Bereich, der in Artikel 17 der neuen Notariatsordnung geregelt wird. Vgl. auch das Stichwort "huibi“ in: Falü Cidian, Shanghai, 1980, S. 251.

$50 \mathrm{Vgl}$. Artikel 23 der neuen Notariatsordnung. 


\section{Die konkreten Aufgaben der Notariate}

Die Notariate werden einmal im "Innenverkehr" (guonei gongzheng shiwu) tätig, d. h. sie führen Beurkundungen und Beglaubigungen für den Gebrauch innerhalb Chinas durch, zum anderen erledigen sie Beurkundungen und Beglaubigungen für den "Außenverkehr" (shewai gongzheng shiwu). Letzteres betrifft Angelegenheiten, in deren Zusammenhang Beurkundungen für die Verwendung im Ausland erforderlich werden. Die Arbeit für den Außenverkehr bildete bisher (auch bereits vor der 'Kulturrevolution`) die Hauptlast der Arbeit der Notariate. Der Verkehr mit dem Ausland war sicher auch ein wesentlicher Beweggrund für die Entscheidung, die Arbeit der Notariate wieder zu normalisieren und endlich fest im Rechtssystem zu verankern. Bei den Millionen von Auslandschinesen, ${ }^{51}$ die Verwandte in China haben und mit diesen Beziehungen aufrechterhalten, wird in einer Vielzahl von Fällen, z. B. bei Personenstandsfragen, Heiraten, Erbschaften, Todesfällen, Adoptionen, Verfügungen über Eigentum usw. eine rechtliche Klärung und in Verbindung damit eine notarielle Beurkundung erforderlich, denn die Behörden der Gastländer verlangen Urkunden und Dokumente. In den letzten Jahren gehen außerdem in größerer Zahl chinesische Praktikanten, Studenten, Austauschwissenschaftler oder Vertreter von Außenhandelsorganisationen ins Ausland, um sich dort länger aufzuhalten. Auch von ihnen verlangen die ausländischen Behörden die Vorlage von beglaubigten Zeugnissen und Urkunden der verschiedensten Art. Hinzu kommt, daß die Zahl der in China residierenden Ausländer ständig zunimmt, ihre rechtlichen Angelegenheiten erfordern unter Umständen notarielle Beurkundungen. ${ }^{51 a}$ Eine zunehmende wirtschaftliche Aktivität Chinas im Ausland ${ }^{51 b}$ führt ebenfalls zur Einschaltung der Notariate, z. B. bei der Beglaubigung von Handelsmarken, Vollmachten, Lizenzen u. a.

In den letzten Jahren spielte die Arbeit im Innenverkehr jedoch eine immer wichtigere Rolle, weshalb gerade der verstärkte Ausbau dieses bisher vernachlässigten Bereiches gefordert wird..$^{52}$ Besonders wichtig im Innenverkehr sind die sogenannten Wirtschaftsverträge (jingji hetong). Derartige Verträge, z. B. über Materiallieferungen, Auftragsfertigung, Transportaufträge, Ratenzahlungskäufe, Kredite usw., deren Abfassung inzwi-

51 Die Auslandschinesen und deren Verwandte werden in verschiedene Gruppen unterteilt: (a) Huaqiao (im Ausland lebende Chinesen); (b) Qiaojuan (in China lebende Verwandte von Auslandschinesen); (c) Guiqiao (nach China zurückgekehrte Auslandschinesen); (d) Gang-Ao tongbao (Landsleute aus Hongkong und Macau) vgl. GM 15. 5. 1981. Außer diesen vier Gruppen gibt es noch die "Waiji huaren ", d. h. Chinesen mit ausländischer Staatsangehörigkeit.

5la Zur rechtlichen Stellung der Ausländer in China vgl. RM 29. 2. 1980, S. 5.

5lb Unter Bezugnahme auf einen Bericht in RM 1. 12. 1982 berichtet AFP (1. 12. 1982), da ßim September 1982 ca. 25 000, Ende November 1982 ca. 30000 chinesische Staatsbürger im Ausland beschäftigt waren: "Peking schloß in den letzten drei Jahren mit 808 ausländischen Unternehmen Kontrakte im Wert von 1,2 Millarden Dollar für den Einsatz chinesischer Ingenieure, Techniker und Facharbeiter ab. Der größte Teil der Projekte im Straßen-, Brücken- und Hafenbau wird im Nahen Osten ausgeführt." Bisher sind 23 chinesische Gesellschaften ermächtigt, Verträge über die Bereitstellung von Gastarbeitern abzuschließen.

52 GM 1. 12. 1981 
schen durch Gesetz geregelt ist, ${ }^{53}$ nehmen im Zuge der Liberalisierung und Belebung des innerchinesischen Wirtschaftsverkehrs stark an Zahl zu. ${ }^{54}$ Vertragspartner sind in der Regel staatliche Betriebe, Kollektivbetriebe oder landwirtschaftliche Produktionsbrigaden, in wechselnder Kombination. Mit der Zunahme der Zahl derartiger Verträge beobachtete man auch eine Zunahme der Vertragsstreitigkeiten, gerade bei Verträgen, die nicht durch Notariate beurkundet waren. Gründe sind u. a. unklare Regelung der Verantwortlichkeiten und der Haftung bei Vertragsverletzung oder verworrene Vertragssprache. ${ }^{55}$ Wenn die Vertragsparteien die notarielle Beurkundung beantragen, sind die Notariate gehalten, vorher eine Prüfung durchzuführen, ob Quantität, Qualität, Preis, Fertigungszeitraum der Waren sowie die wirtschaftlichen Voraussetzungen der Vertragsparteien (Anlagen, Technologie, Personal) zuverlässig sind, wie die Kosten verteilt werden, wie die Haftung bei Vertragsverletzung oder Nichterfüllung geregelt wird, welches die Bedingungen für die Änderung oder Auflösung der Verträge sind. ${ }^{56}$ Die notarielle Beurkundung hat rechtlich bindende Wirkung und soll die Rechte der Vertragsparteien schützen. In zunehmendem Maß werden auch Verträge mit ausländischen Partnern, z. B. bei Joint-Venture-Betrieben in China, von den Notariaten beurkundet. ${ }^{57}$

Auch Beurkundungen für den Außenverkehr haben einen wirtschaftlichen Aspekt, der für den chinesischen Staat in der heutigen Zeit von einigem Interesse ist, ${ }^{58}$ denn hierbei geht es oft um Eigentums- und Erbschaftsangelegenheiten. Die von den Notariaten in China ausgestellten Urkunden

"erleichtern es den Bürgern unseres Landes, Besitz im Ausland ${ }^{59}$ nach China zurückzuholen, wobei nicht nur die Rechte und Interessen der beteiligten Parteien geschützt werden, sondern auch für den Staat eine nicht unbeträchtliche Menge an nichtkommerziellen Devisen (fei-maoyi waihui) eingebracht wird. So haben (1979) die Notariate der beiden Städte Shanghai und Guangzhou für den Staat 840 000,- Yuan an Devisen zurückgeholt, und die Notariate von 12 Städten und Kreisen des Regierungsbezirks Foshan (Prov. Guangdong) haben über 1,1 Millionen Yuan Devisen zurückgeholt, was eine kraftvolle Unterstützung für den Aufbau im Rahmen der "Vier Modernisierungen " darstellt. ${ }^{60}$

53 Vgl. Fn. (10); dt. bei: F. Münzel, "Zum Wirtschaftsvertragsgesetz der VR China vom 13. 12. 81 ", in: WGO 1981 , S. $151 \mathrm{ff}$.

54 Die Volksgerichte haben inzwischen begonnen, eigene Wirtschaftskammern (jingji shenpanting) einzurichten.

55 Beispiele hierfür in GM 1. 12. 1981.

56 GM 1. 12. 1981.

57 Besonders bei den Notariaten in Peking und Kanton (Guangzhou).

58 GM 15. 8. 1980.

59 Im Chinesischen wird hier der Begriff "yuwai" (wörtl. "außerhalb des Gebietes") verwendet, also raußerhalb der Landesgrenzenc. Dadurch wird das Wort Ausland vermieden, um auch Territorien, die die Volksrepublik China nicht als Ausland ansieht, wie Hongkong und Macao, einzubeziehen.

60 GM 15. 8. 1980, vgl. auch Beijing Ribao (BJ) 18. 4. 1981 und Falü Shouce (1979), S. 54. GM 15. 8. 1980 schildert einen Fall, den das Notariat in Kunming (Provinz Yunnan) im Jahr 1980 zu bearbeiten hatte. Es ging um eine Erbschaft außerhalb der VR China. Das Notariat entsandte dreimal Mitarbeiter nach Guangzhou, um die Fakten zu prüfen, um sich über Zivilprozeß-Regelungen in Hongkong zu informieren, um mit Leuten, die die Umstände kannten, Kontakt auf zunehmen und um Experten, die sich im Hongkonger Rechtssystem auskannten, zu bef ragen. Dadurch sollten die Vor- und Nachteile eines Prozesses bzw. eines nicht-prozessuralen Vorgehens in dieser Angelegenheit geklärt werden. Die beteiligten Parteien wurden überredet, die Angelegenheit in China zu regeln, und so wurden über 700000 Yuan an inichtkommerziellen Devisen zurückgeführt. 
Faßt man die konkreten Aufgaben der Notariate zusammen, die in verschiedenen Quellen genannt werden (auch bereits vor der Verabschiedung der Notariatsordnung), so ergibt sich eine Liste von Beurkundungen oder Beglaubigungen in folgenden Angelegenheiten: ${ }^{61}$

(1) Wirtschaftsverträge (jingji hetong, auch qiyue genannt) zwischen chinesischen Partnern $^{62}$

(2) Wirtschaftsverträge zwischen chinesischen und ausländischen Partnern ${ }^{63}$

(3) Eintragung von Handelsmarken (shangbiao zhuce), Lizenzen (yingye zhengshu), Beglaubigung von Testberichten (shiyan baogao), Garantien (danbao), Vollmachten (shouquan), Bescheinigungen über gegenseitige Begünstigung (huhui zhengming $)^{64}$

(4) Vollmachten (weituoshu) $)^{65}$

(5) Adoptionen (shouyang guanxi) ${ }^{66}$

(6) Immobilienkauf und -verkauf (fangwu maimai) ${ }^{67}$

(7) Vermietungen (zulin) $)^{68}$

(8) Testamente (yizhu $)^{69}$

(9) Erbschaften (xucheng) ${ }^{70}$

61 Diese Auf zählung erhebt keinen Anspruch auf Vollständigkeit. Auch die Auf zählung in Artikel 4 der neuen Notariatsordnung nennt nur die wichtigsten Bereiche notarieller Tätigkeit. Erschwert wird ein klares Bild durch unterschiedliche Benennung der einzelnen Arten von Beurkundung sowie das Fehlen ausführlicher Erklärungen dazu.

62 GM 3. 7. 1981.

63 BJ 18. 4. 1981.

64 Diese Beurkundungen, vorgenommen fü $r$ die Zentralregierung und fü $r$ Betriebe der Stadt Peking, werden vom Notariat Peking berichtet, vgl. BJ 18.4. 1981. Sie stehen im Zusammenhang mit chinesischen Wirtschaftsaktivitäten im Ausland (Bauprojekte, Teilnahme an internationalen Ausschreibungen, Kreditvergabe, Joint Ventures, Außenhandelsgeschäfte) und sollen "die Rechte und Interessen Chinas bei seinen außenwirtschaf tlichen Aktivitäten schützen".

65 RM 3. 10. 1980; GM 15. 5. 1981; GM 3. 7. 1981.

66 G M 15. 5. 1981; B J 18. 4. 1981. Uber Einzelheiten des Adoptionsverfahrens gibt u. a. ein Buch Auskunft, das nach der Verkündung der neuen Notariatsordnung erschienen ist: Feng Ertai u. a., Falü Wenda Sibai Ti (400 Fragen und Antworten zum Recht), Shanghai: Xuelin Chubanshe, 1982, S. 319-320. Die Adoption ist im allgemeinen beschränkt auf noch nicht erwachsene Kinder, die Zustimmung beider leiblichen Elternteile ist erforderlich. Die Beurkundung wird durch die Notariate vorgenommen. Das Adoptiv-Verhältnis kann ohne Erlaubnis nicht von irgendeiner Seite gelöst werden, dies ist nur bei Zustimmung beider Parteien möglich. In Streitfällen entscheiden die Volksgerichte. Die Adoption erfolgt auf freiwilligen Antrag der Adoptiveltern wie der leiblichen Eltern. Falls die adoptierte Person bereits geschäftsfähig ist (you xingwei nengli), muß sie auch ihre Zustimmung geben, sie darf nicht durch Drohung, Zwang oder eine versteckte Form des Verkaufs zur Adoption gebracht werden. Wenn die Adoptiveltern in großen Städten wohnen, die Familie der zu adoptierenden Person aber auf dem Lande bzw. außerhalb, dann müssen beide Parteien zunächst bei den Sicherheitsbehörden Formalitäten hinsichtlich der Wohnsitzf rage regeln, bevor die notarielle Beurkundung durchgeführt wird. Vgl. auch Yang Dawen (Hrsg.) Hunyinfa Jiaocheng (Kurs zum Eherecht), Peking: Falü Chubanshe, 1982, S. $227 \mathrm{ff}$.

67 GM 15. 5. 1981; GM 5. 5. 1981.

68 Falü Cidian, Shanghai, 1980, S. 126.

69 RM 3. 10. 1980; GM 15. 5. 1981; GM 3. 7. 1981.

70 R M 3. 10. 1980 (caichan xucheng); GM 15. 5. 1981; B J 18. 4. 1981; zum Erbschaftsrecht vgl. auch R M 29. 6. 1980, S. 5. 
(10) Identität/Status (shenfen) ${ }^{71}$

(11) Personenstandsangelegenheiten, z. B.

- Verwandtschaftsbeziehungen (qinshu guanxi) ${ }^{72}$

- Geburten (chusheng) ${ }^{73}$

- Heiraten (hunyin zhuangkuang) ${ }^{74}$

- Scheidungen (lihun) ${ }^{75}$

- Todesfälle (siwang) ${ }^{76}$

- Verschollenheit (shizong) ${ }^{77}$

- Lebenszeugnisse (shengcun) ${ }^{78}$

- Staatsangehörigkeit (guoji) ${ }^{79}$

(12) Studiengänge (xueli), ${ }^{80}$ Arbeitsverhältnisse/Arbeitserfahrungen (jingli) ${ }^{81}$

(13) eidesstattliche Erklärungen (shengmingshu) ${ }^{82}$

(14) Úbereignungen (caichan zhuanrang) ${ }^{83}$

(15) Besitzteilung (caichan fenshu) ${ }^{84}$

(16) Familienteilungen (fenjia) ${ }^{85}$

(17) Familienvereinbarungen (jiating xieyishu) $)^{86}$

(18) Aufbewahrung von Dokumenten (baoguan wenju), ${ }^{87}$ einschließlich der Aufbewahrung von Testamenten ${ }^{87 a}$

71 RM 3. 10. 1980.

72 RM 3. 10. 1980; GM 15. 5. 1981.

73 GM 15. 5. 1981; GM 3.7. 1981.

74 R M 3. 10. 1980; GM 15. 5. 1981. Die Original-Heiratsurkunden werden in der Regel von den Standesämtern (hunyin dengjichu) ausgestellt, vgl. Nr. 7 Hunyin dengji banfa, in: Guowuyuan Gongbao, Nr. 347, S. 582 f.; dt. bei: W. Keßler, Das Standesamt, 6-7/82, S. $195 \mathrm{ff}$.

Bei Heiratsurkunden können die Notariate in folgender Weise tätig werden: Ist eine Originalurkunde vorhanden, so kann die Echtheit der Urkunde vom Notariat noch einmal beglaubigt werden. Ist die Originalurkunde verloren gegangen, so stellt das Notariat Nachforschungen bei den Standesämtern der Orte an, wo die Hochzeit jeweils stattgef unden hat, oder erkundigt sich in den Herkunftsorten der Partner. Kommt es zur Ausstellung einer Urkunde, so ist diese ein Ersatz-Dokument. Ist eine Heiratsurkunde nie ausgestellt worden (vorwiegend nur bei heute alten Leuten der Fall), so müssen die Notariate versuchen, anhand "faktischer Beweise“, z. B. dem Vorhandensein von Kindern, die Eheschließung festzustellen und darüber eventuell eine Urkunde auszustellen. Von da dürften auch Ehefähigkeitszeugnisse nach $\S 10$ Abs. 1 Ehegesetz-BRD zu beschaffen sein.

75 GM 28. 3. 1980.

76 RM 3. 10. 1980; GM 15. 5. 1981; GM 3. 7. 1981.

77 RM 3. 10. 1980.

78 RM 3. 10. 1980; RM 18. 12. 1979.

79 GM 15. 5. 1981.

80 RM 3. 10. 1980; GM 15. 5. 1981; GM 3. 7.1981.

81 RM 3. 10. 1980; GM 3. 7. 1981, z. B. die notariell beglaubigte Feststellung, da $§$ und als was eine bestimmte Person zu einer bestimmten Zeit in einem bestimmten Betrieb gearbeitet hat.

82 Z. B. in Fällen, wo über stattgefundene Rechtsgeschäfte oder rechtlich relevante Tatsachen keine Urkunden mehr vorhanden sind.

83 GM 15. 5. 1981.

84 GM 5. 5. 1981.

85 B J 18. 4. 1981; d.h. Vereinbarungen über Verteilung von Familienvermögen a n erwachsene Kinder.

86 GM 28. 9. 1980. Es handelt sich um Vereinbarungen über die Aufteilung der Lasten, die in einer Familie bei der Versorgung der alten Leute entstehen.

87 Falü Cidian, Shanghai, 1980, S. 126. 
(19) Sicherung von Beweismitteln (baoguan zhengju) ${ }^{88}$

(20) Nachlaßverwaltung (yichan de baoguan) ${ }^{89}$

(21) Beglaubigung der Ubereinstimmung mit dem Original von

- Kopien (fuben) ${ }^{90}$

- Auszügen (jieben $)^{91}$

- Ubersetzungen (yiben) ${ }^{92}$

- Ablichtungen (yingyinben) ${ }^{93}$

- Siegeln und Unterschriften (qianming gaizhang) ${ }^{94}$

(22) Grundstücksangelegenheiten ${ }^{95}$

(23) Wohnsitzfragen (juzhu) ${ }^{96}$

(24) vollstreckbare Urkunden ${ }^{97}$

(25) Angelegenheiten im Zusammenhang mit Pensionszahlungen für zurückgekehrte Auslandschinesen ${ }^{98}$

(26) Schenkungen (zengyu) ${ }^{99}$

(27) Vermächtnisse (yizeng) $)^{100}$

88 GM 28. 3. 1980; Falü Cidian, S. 126.

89 GM 28. 3. 1980.

90 RM 3. 10. 1980; GM 3. 7. 1981.

91 RM 3. 10. 1980.

92 RM 3. 10. 1980; GM 3. 7. 1981.

93 RM 3. 10. 1980.

94 RM 3. 10. 1980.

95 Da der Grund und Boden in China im wesentlichen in Gemeineigentum überführt wurde, spielen Grundstücksgeschäfte, vor allem private, kaum noch eine Rolle. In jüngster Zeit wurden jedoch Fälle bekannt, in denen man zur Regelung von Grundstücksangelegenheiten die Notariate wieder heranzog (RM 27. 9. 1982): in der im Kreis Changping (ein Landkreis außerhalb Pekings) gelegenen Produktionsbrigade Baihe gab es fortwährend Streit um die Abgrenzung von Baugrundstücken für neue Bauernhäuser. Mit offenbar steigendem Wohlstand hatte die Bautätigkeit in der Brigade (252 Familien mit insgesamt 1020 Personen) stark zugenommen, bis Januar 1982 bauten sich 147 Familien neue Wohnhäuser. Aus vielerlei "historischen Gründen" (die Quelle schweigt über die Art der Gründe), gab es zwischen benachbarten Familien bzw. zwischen einzelnen Personen und dem Kollektiv fortwährend Streit wegen unklarer Grundstücksgrenzen. Von den 82 Zivilstreitigkeiten im 1. Halbjahr 1982 betrafen allein 70 (85,37\%) die Hausgrundstücke. Die Justizbehörden sahen sich genötigt, durch die Entsendung von Funktionären einzugreif en, worauf immerhin 14 Fälle geschlichtet und 10 Fälle "geregelt" werden konnten, 4 Fälle blieben ungelöst. Man hielt es für wirksam, Fläche und Nutzungsrecht der Grundstücke festzulegen und von den Justizbehörden beglaubigen zu lassen. Im Juli 1982 wurde Notariatspersonal in die Brigade geschickt, das für 145 Familien die Grenzen der Hausgrundstücke festlegte und 172 notarielle Beurkundungen vornahm.

96 RM 18. 12. 1979.

97 RM 3. 10. 1980. Auf Dokumenten, die die Rückforderung von Darlehen oder Sachen betreffen können die Notariate, wenn die Sachlage eindeutig ist, die Vollstreckbarkeit beurkunden. Vgl. auch Falü Dawen Sibai Ti, a.a.O., S. 318.

98 BJ 18. 4. 1981.

99 GM 15. 5. 1981.

100 GM 15. 5. 1981. 


\section{Statistische Angaben zur Arbeit einiger ausgewählter Notariate}

Ein wenig anschaulicher wird das Bild, wenn man sich die Arbeit einiger Notariate in chinesischen Großstädten während der letzten Jahre ansieht. Hierbei wird auch die rasche Aufwärtsentwicklung deutlich.

Nach neuesten Berichten ${ }^{101}$ besitzt die Stadt Peking heute bereits 17 Notariate, einschließlich der Notariate in den Stadtbezirken und den Landkreisen außerhalb der Stadt. Das "Notariat der Stadt Peking" (Beijing Shi Gongzhengchu) ist als einziges Notariat in Peking zuständig für den Außenverkehr, also die Ausstellung von Urkunden zur Verwendung im Ausland (z. B. auch Beglaubigung der Zeugnisse ausländischer Studenten, die an chinesischen Hochschulen studieren). Nach Angaben eines stellvertretenden Leiters des Notariats ${ }^{102}$ hat diese Behörde auch während der 'Kulturrevolution` in stark reduziertem Umfang weitergearbeitet, überwiegend im Außenverkehr. Der Personalbestand für Ende 1981 wurde mit "ca. 30 Personen" (Fachpersonal) angegeben, er sei doppelt so hoch wie 1980. In der Zunahme des Personals spiegelt sich die starke Ausweitung der Nachforschungsarbeiten wider, die das Notariat im Zusammenhang mit der Bearbeitung der bei ihm eingereichten Anträge durchführen muß.

Im Dezember $1979^{103}$ wird vom Pekinger Städtischen Notariat berichtet, daß "in nicht ganz 3 Jahren “ 4799 Beurkundungen für den Außenverkehr vorgenommen wurden, davon in der 1. Hälfte 1979 allein 1737 Beurkundungen. Als Antragsteller werden genannt: Auslandschinesen und deren Verwandte, Landsleute aus Hongkong und Macao, Bürger der Volksrepublik China, die ins Ausland gehen sowie in China residierende Ausländer. Beurkundungen im Innenverkehr sind im Pekinger Städtischen Notariat erst seit der zweiten Jahreshälfte 1980 möglich. ${ }^{104}$ Bis 4 . April 1981 wurden 466 Anträge auf Beurkundung eingereicht, im Jahr 1980 waren es 219 Anträge. ${ }^{105}$ Die Hälfte der Anträge behandelte Wirtschaftsverträge, darunter 158 Ratenzahlungsverträge für den Kauf von Kühlschränken. Für den Außenverkehr erhielt das Notariat im Jahr 19806594 Anträge, es wurden 4292 Urkunden ausgestellt.

Ein Bericht vom November $1982^{106}$ enthält eine "Fortschreibung" der statistischen Angaben bis zu diesem Zeitpunkt: "Beijing's public notary offices, since their resumption of activity in 1980, have handled 4604 domestic cases and 10,007 international cases. " Dies bezieht sich auf die Arbeit aller Notariate in Peking. Die statistischen Angaben sind wegen der ständig wechselnden Bezugsgrößen für ein klares Gesamtbild nur bedingt zu gebrauchen. Das Pekinger Städtische Notariat bearbeitete dem gerade zitierten Bericht zufolge in den letzten beiden Jahren 260 Erbschaftsfälle, 139 Adoptionen, 37 Nachlaß- 
reglungen und 237 Testamente. In einem Fall errichtete ein Notariat in einem Geschäft für Kühlschränke einen Schalter, um insgesamt 3693 Ratenkaufverträge zu beglaubigen, damit die Zahlungen durch die Käufer und die Service-Leistungen durch den Verkäufer gewährleistet wurden. ${ }^{107}$

Das Notariat der Stadt Harbin ${ }^{108}$ hat vom 1. 1. 1980 bis 10.9. 1980 im Innenverkehr 201 Beurkundungen bearbeitet, z. B. Erbschaften, Familienverträge, Testamente, Adoptionen.

Nach unvollständigen Statistiken für 22 Provinzen wurden 1979 für den Außenverkehr fast 150000 Beurkundungen bearbeitet, 1980 (Jan.-Juni) 2500 Fälle im Notariat Shanghai und 2100 Fälle im Pekinger Städtischen Notariat sowie, in der Zeit von April bis Juni 1980, allein 12000 Fälle im Notariat der Stadt Foshan (Provinz Guangdong). ${ }^{109}$ Die jüngsten Zahlen für das gesamte Land ${ }^{110}$ nennen für das Jahr 1981 über $250000 \mathrm{Be}-$ urkundungen, ${ }^{111}$ die in den 1600 Notariaten durchgeführt wurden, darunter über 100000 Urkunden für den Außenverkehr. Betrachtet man den Zeitraum von 1979 bis zum Frühjahr 1982, so ergibt sich etwa folgendes Bild: "According to ministry statistics, since 1979, the nation's notarial offices have registered 600000 documents, more than one-quarter million of them last year (1981) alone. Most have involved the affairs of non-residents or non-Chinese residents . . (Such)documents, according to the statistics, have thus far been presented to 80 nations and regions. " 112

\section{Formvorschriften}

Haben die Notariate nach Abschluß ihrer Nachforschungen dem Antrag auf Beurkundung stattgegeben, müssen sie in vorgeschriebener Form Urkunden ausstellen (gongzhengshu). Artikel 20 und 21 der neuen Notariatsordnung regeln summarisch die Ausstellung dieser Dokumente, deren Form vom Justizministerium festgelegt oder genehmigt wird. Úber die Formvorschriften im einzelnen sind an anderer Stelle Details bekannt geworden, ${ }^{113}$ die den Schluß nahelegen, daß in der Praxis bereits bestimmte Regelungen bestanden. ${ }^{114}$

Auf den Urkunden muß der Name des Notariats und dessen Dienstsiegel sowie Unterschrift ${ }^{115}$ oder Namenssiegel des Notars vorhanden sein. Da die Urkunden Dokumenten

China Daily, a.a.O.

GM 28. 9. 1980.

GM 15. 8. 1980.

RM 29. 6. 1982.

RM 25. 4. 1982 konkretisiert diese Zahl etwas: über 251,000 Fälle.

Xinhua (Engl.), 23. 4. 1982.

Tang/Yang/Cheng/Zhu, ... (Grundwissen), oben Fn. (46), S. 245-247.

Es handelt sich wahrscheinlich, wie so oft in der chinesischen Verwaltung, um interne Richtlinien, die in der Offentlichkeit nicht bekanntgegeben werden.

5 Bei den dem Verfasser zu Gesicht gekommenen Urkunden war die Unterschrift des Notars stets durch einen Faksimile-Stempel der Unterschrift in blauer Farbe angebracht worden. 
von "wichtiger Beweiskraft"116 sind bzw. häufig auch für die Verwendung im Ausland bestimmt sind, soll die Ausfertigung sehr verantwortungsbewußt und sorgfältig vorgenommen werden, um ihre Qualität zu garantieren.

Bei der Ausfertigung der Urkunden sind folgende Punkte zu beachten:

(1) Beurkundungen müssen entsprechend den rechtlich festgelegten Inhalten und Verfahren vorgenommen werden. Notwendige Recherchen müssen sorgfältig durchgeführt werden um zu garantieren, daß die notariellen Urkunden wahrheitsgemäß und juristisch einwandfrei sind.

(2) Jeweils für einen Sachverhalt wird eine Urkunde ausgestellt. Der Inhalt einer notariellen Urkunde bezieht sich im allgemeinen nur auf einen Vorgang.

Wenn mehrere Sachverhalte, bezüglich derer ein Antrag auf Beurkundung gestellt wird, ein und denselben Verwendungszweck haben, können mehrere Vorgänge zusammen auf einer Urkunde beurkundet werden.

(3) Altersangaben (Geburtsdaten) in den notariellen Urkunden sollen einheitlich nach westlichem Kalender geschrieben werden. Im Bedarfsfall kann in Klammern dahinter die Angabe nach dem chinesischen (Mond-)Kalender erfolgen.

(4) Bei der Angabe von Geburtsorten werden im allgemeinen nur die Provinz (bzw. das Autonome Gebiet oder die Regierungsunmittelbare Stadt) sowie die Namen der Kreise und Städte genannt, um zu vermeiden, daß durch Änderung des konkreten Ortsnamens Unannehmlichkeiten entstehen. Falls sich diese Namen geändert haben, muß der frühere Name unter Voranstellung des Wortes "früher" geschrieben werden, um so den Unterschied zu verdeutlichen.

(5) Wenn die Urkunden vom Antragsteller unterzeichnet werden müssen, sollen sie in der Regel unterschrieben werden. Wer nicht schreiben kann, kann ein Siegel verwenden. ${ }^{117}$ Die Unterschrift darf nicht durch einen Fingerabdruck ersetzt werden. Wenn der Notar Unterschrift und Siegel des Antragstellers beglaubigt, muß der Bezug (d. h. was jeweils beglaubigt wird) klar sein.

(6) Auf der Mehrzahl der Urkunden braucht keine Fotografie angebracht zu werden. Bei Urkunden über Berufserfahrungen/Berufstätigkeiten (jingli zhengmingshu) oder Bescheinigungen über ein Studium (xueli zhengmingshu), die als materielle Urkunden verwendet werden, sollte ein Foto beigefügt werden. Heirats- und Geburtsurkunden können mit Foto versehen werden, wenn der Antragsteller einen legitimen Grund angibt.

(7) Der Inhalt der Urkunden muß wohlgeordnet und klar sein, streng logisch, die Sprache verständlich und einfach. Die sprachliche Formulierung soll nach der Standardsprache vorgenommen werden, es dürfen keine schwerverständlichen Dialektaus-

116 Einige Urkunden können direkt wirksam werden, d. h. ihre Vollstreckung wird von Vollstreckungsbeamten der Volksgerichte verantwortlich überwacht.

117 Namens-Siegel sind in China allgemein gebräuchlich. Ihre Form kann frei gewählt werden, z. B. unter Verwendung archaischer Formen von Schriftzeichen. Die Siegel werden auf Dokumenten als gleichwertig mit Unterschriften anerkannt. Für die Namens-Siegel muß rote Farbe verwendet werden. 
drücke verwendet werden, desgleichen keine vereinfachten Schriftzeichen, die noch nicht vom Staatsrat öffentlich verkündet worden sind.

Die Wirksamkeit der Urkunden beginnt mit der Unterzeichnung durch den Notar. ${ }^{118}$ Dokumente, die außerhalb Chinas verwendet werden, müssen unter Umständen noch durch das Außenministerium der Volksrepublik China bzw. die Konsularabteilungen der Botschaften des Landes, in dem die Urkunden verwendet werden, legalisiert (renzheng) werden. Die Urkunden werden in einem solchen Fall am Tage der Legalisation wirksam. ${ }^{119}$ Fehlerhaft ausgestellte Urkunden müssen, sobald dies festgestellt wird, von den Notariaten oder den zuständigen Justiz-Verwaltungen ungültig gemacht werden.

\section{Zukunftsperspektiven}

Wie die jüngsten Angaben belegen, ${ }^{120}$ hat die Zahl der bisher eingerichteten Notariate den Stand von 1957 bereits überschritten. Eine Aufstellung über die Standorte ${ }^{121}$ der Notariate ist nicht möglich. Die Notariatsordnung von 1982 - obwohl als vvorläufigı bezeichnet - setzt nun einen rechtlichen Rahmen für Aufbau und Aufgaben der Notariate, die Planung des weiteren Ausbaus obliegt den Justizverwaltungs-Behörden. Eine Gebührenordnung steht offenbar noch aus bzw. es ist möglich, daß man hier nach den bisher angewandten internen Verwaltungsrichtlinien, die nicht im einzelnen bekannt sind, verfährt. Der Ausbau des Notariatswesens wird weiter gehen, dieses Ziel wurde jüngst wieder auf einer nationalen Konferenz über politische und juristische Arbeit in Peking bekräftigt. ${ }^{122}$ Fachprobleme aus dem Bereich der Notariate werden offenbar auch in die juristische Ausbildung ${ }^{123}$ mit einbezogen. ${ }^{124}$ Besondere Aufmerksamkeit wird neben der Ausweitung des Personalbestandes und einer Verbesserung der Ausbildung in Zukunft dem Bereich Innenverkehr zu schenken sein, der im Verhältnis zu den notariellen Aktivitäten im Außenverkehr stark vernachlässigt wurde. Gerade für eine geordnete Entwicklung der Volkswirtschaft wünscht man mehr rechtliche Klarheit. Bereits 1979 forderte ein Autor, ${ }^{125}$ in ein zukünftiges Zivilgesetzbuch ${ }^{126}$ Bestimmungen darüber aufzunehmen, welche Rechtshandlungen, welche Dokumente und Tatsachen von rechtlicher Bedeutung

118 GM 3. 7. 1981

119 GM 3. 7. 1981, desgl. Tang/Yang/Cheng/Zhu, ... (Grundwissen), oben Fn. (46), S. 245.

120 Vgl. RM 25. 4. 1982.

121 Dem Verfasser bekanntgeworden sind z. B. die Notariate der Städte Peking, Shanghai, Harbin, Guangzhou, Baoding (Prov. Hebei), Foshan (Prov. Guangdong), des Kreises Xinhui (Prov. Guangdong), des Kreises Qingtian (Prov. Zhejiang), des Regierungsbezirks Ningbo (Prov. Zhejiang).

122 10.-24. Juli 1982, vgl. RM 25. 7. 1982; auch China aktuell, Juli 1982, S. 380.

123 China besaß Anfang 1982 vier Hochschulen für Politik und Recht sowie 21 juristische Abteilungen an Hochschulen, mit insgesamt mehr als 9000 Studenten, vgl. Xinhua (Englisch) 31. 3. 1982.

124 Dies wurde dem Verfasser beim Besuch im Pekinger Notariat (1981) angedeutet. Es hieß, man plane auch die Einrichtung einer eigenen Studienf achrichtung (unklar wo und wie).

125 RM 18. 12. 1979.

126 Im August 1980 wurde bereits ein "Diskussions-Manuskript" fertiggestellt, vgl. GM 12. 6. 1981. Bisher ist das Zivilgesetzbuch noch nicht verabschiedet worden. 
notariell beurkundet werden müssen, um rechtliche Wirksamkeit zu erlangen. Ein solcher Zwang würde die Parteien zu mehr Sorgfalt beim Vertragsschluß und zur gewissenhaften Einhaltung geschlossener Verträge zwingen. Eine zukünftige Zivilprozeßordnung $^{127}$ sollte außerdem klare Bestimmungen über Schuldurkunden (zhaiwu wenshu) enthalten. ${ }^{128}$ Gerade bei den Wirtschaftsverträgen, deren Abfassung und Durchführung inzwischen gesetzlich geregelt ist, ${ }^{129}$ kommt den Notariaten eine Kontrollfunktion (gongzheng jiandu) zu, die sie vermittels der von ihnen durchgeführten Prüfung und öffentlichen Beurkundung ausüben. ${ }^{130}$

Wenngleich die zuweilen über das Notariatswesen im Ausland, insbesondere in den kapitalistischen Ländern geäußerten Ansichten, ${ }^{131}$ unter etwas einseitigem politischen Gesichtswinkel dargeboten werden, so hat man es doch nicht versäumt, als einschlägiges ausländisches Gesetz zum Notar-Recht das Japanische Notar-Gesetz ${ }^{132}$ ins Chinesische zu übersetzen, als von außen kommende Bereicherung der Diskussion.

\section{Vorläufige Notariatsordnung für die Volksrepublik China}

(Verkündet vom Staatsrat am 13. April 1982)

\section{Abschnitt I: Allgemeine Regeln}

\section{Artikel 1}

Um das System der staatlichen öf fentlichen Beurkundung auszubauen, das sozialistische Rechtssystem zu schützen, Streitfällen vorzubeugen und die Zahl der Prozesse zu verringern, werden die nachfolgenden Bestimmungen erlassen.

127 Die am 8. 3. 1982 vom Nationalen Volkskongreß verabschiedete Zivilprozeßordnung trat am 1. 10.1982 in Kraft. Text in GM 11. 3. 1982, englische Ubersetzung in Summary of World Broadcasts, Part III, 17. 3. 1982; dt. Ubers. v. F. Münzel, RabelsZ (März) 1983), in Vorbereitung.

128 Die neue ZPO enthält keine Vorschriften über derartige Fälle, dafür aber Artikel 24 der neuen Notariatsordnung.

129 Zhonghua Renmin Gongheguo Jingji Hetong Fa, Text in Guowuyuan Gongbao (Bulletin des Staatsrates) Nr. 373/1981 S. 864-876. Hintergrundwissen über Wirtschaftsverträge vermittelt Li Zhuguo und Bai Youzhong, Hetong Jiben Zhishi (Grundwissen über Verträge), Peking: Qunzhong Chubanshe, 1981; Jingji Hetong Xuanbian (Auswahl von Rechtsvorschriften zu Wirtschaftsverträgen), Peking: Gongren Chubanshe, 1981.

Das Gesetz über Wirtschaftsverträge enthält keine expliziten Vorschriften darüber, welche Verträge notariell beurkundet werden müssen und welche nicht. Dies wird von den zuständigen Wirtschaftsverwaltungsbehörden (z. B. Wirtschaftskommission, Verwaltungsämter für Industrie und Handel) der Provinzen, Städte und Autonomen Gebiete entsprechend den konkreten Verhältnissen in den jeweiligen Gebieten in eigener Verantwortung bestimmt. Vgl. dazu Wang Zhong u. a., Jingji Faxue (Wirtschaftsrecht), Changchun: Jilin Renmin Chubanshe, 1982, S. 130.

130 Vgl. Liu Longheng, Jingjifa Jianlun (Kurzer Abriß des Wirtschaftsrechts), Peking: Beijing Daxue Chubanshe, 1981, S. 115, sowie Wang Zhong, a.a.O. S. 129. Zunächst üben natürlich die für die jeweilige Wirtschaftssparte zuständigen Verwaltungsbehörden, bei denen die Verträge registriert werden, die Úberwachung aus. Auch die Banken, die u. Umständen Kredite geben, haben eine Kontrollfunktion.

131 RM 18. 12. 1979.

132 Riben Gongzhengren Fa (Das Japanische Notar-Gesetz), Peking: Falü Chubanshe, 1981. 


\section{Artikel 2}

Offentliche Beurkundung (gongzheng) ist die von den Notariatsorganen des Staates auf Antrag der Beteiligten entsprechend den Gesetzen vorgenommene Beurkundung von Rechtsgeschäften sowie der Echtheit und Rechtmäßigkeit von rechtlich relevanten Dokumenten und Tatsachen mit dem Ziel, das öffentliche Eigentum sowie die Rechte und legalen Interessen der Bürger bezüglich ihres Status und ihres Eigentums zu schützen.

\section{Artikel 3}

Die Notariate (gongzhengchu) sind Organe des Staates für öffentliche Beurkundung. Die Notariate sollen vermittels ihrer notariellen Tätigkeit die Bürger zur Einhaltung der Gesetze erziehen und das sozialistische Rechtssystem schützen.

\section{Abschnitt II: Die Aufgaben der Notariate}

\section{Artikel 4}

Die Notariate haben die folgenden Aufgaben:

(1) Die Beurkundung von Verträgen, Vollmachten und Testamenten;

(2) die Beurkundung von Erbschaftsrechten;

(3) die Beurkundung der Schenkung oder Teilung von Eigentum;

(4) die Beurkundung von Adoptivbeziehungen;

(5) die Beurkundung von Verwandtschaftsbeziehungen;

(6) die Beurkundung der Identität, von Studiengängen und von Arbeitserfahrungen;

(7) die Beurkundung von Geburten, ehelichen Verhältnissen, Lebenszeugnissen und Todesfällen;

(8) die Beurkundung der Echtheit von Unterschriften und Siegeln auf Dokumenten;

(9) die Beurkundung der Ubereinstimmung mit dem Original von Abschriften, Auszügen, Úbersetzungen und Ablichtungen von Dokumenten;

(10) bei Dokumenten über die Rückforderung von Darlehen oder Sachen die Beurkundung, sofern keine Zweifel bestehen, auf den Dokumenten selbst, daß aus diesen vollstreckt werden kann;

(11) die Sicherung von Beweismitteln;

(12) die Aufbewahrung von Testamenten oder anderen Urkunden;

(13) die Vertretung der Beteiligten bei der Abfassung von Unterlagen für die Beantragung einer notariellen Beurkundung;

(14) die Durchführung anderer notarieller Aufgaben entsprechend den Anträgen der Beteiligten bzw. entsprechend den internationalen Gepflogenheiten.

\section{Abschnitt III: Organisation und Leitung der Notariate}

\section{Artikel 5}

In. den Regierungsunmittelbaren Städten, Landkreisen (Autonomen Kreisen) und Städten werden Notariate eingerichtet. Mit Genehmigung der Justizverwaltungsbehörden der Provinzen, Autonomen Gebiete und Regierungsunmittelbaren Städte können auch in Stadtbezirken Notariate eingerichtet werden.

\section{Artikel 6}

Die Notariate stehen unter Leitung der Justizverwaltungsbehörden. Zwischen den Notariaten gibt es keine Unterordnungsverhältnisse.

\section{Artikel 7}

An den Notariaten gibt es die Stellen des Notars und des assistierenden Notars. Nach Bedarf können auch die Stellen des Leiters und des stellvertretenden Leiters eingerichtet werden.

Leiter und stellvertretende Leiter werden aus dem Kreis der Notare berufen. Leiter und stellvertretende Leiter leiten die Arbeit der Notariate, darüber hinaus müssen sie ihren Beruf als Notar ausüben. 
Leiter, stellvertretende Leiter, Notare und assistierende Notare werden jeweils von den Volksregierungen der Regierungsunmittelbaren Städte, der Kreise und der Städte nach den entsprechenden Verwaltungsvorschriften für Funktionäre ernannt und entlassen.

\section{Artikel 8}

Bürger, die das aktive und passive Wahlrecht besitzen und eine der nachfolgend aufgeführten Bedingungen erfüllen, können zum Notar berufen werden:

(1) Erfolgreiche Absolventen der juristischen Fachrichtungen der Hochschulen, die mehr als ein Jahr Erfahrung in der Justizarbeit, der juristischen Ausbildungsarbeit oder der juristischen Forschung haben;

(2) Personen, die an Volksgerichten oder Volksstaatsanwaltschaften das Amt des Richters oder Staatsanwalts ausgeübt haben;

(3) Personen, die mehr als zwei Jahre Erfahrung in der Justizarbeit bei den Justizverwaltungsbehörden haben oder die mehr als fünf Jahre Arbeitserfahrung bei anderen staatlichen Organen, Verbänden, Betrieben und Institutionen haben und darüber hinaus entsprechende juristische Fachkenntnisse vom Niveau der Absolventen juristischer Sekundarschulen haben;

(4) Personen, die mehr als zwei Jahre das Amt eines assistierenden Notars innegehabt haben.

\section{Artikel 9}

Erfolgreiche Absolventen juristischer Hoch- oder Sekundarschulen sowie Staatsfunktionäre mit gleichwertiger Ausbildung können zum assistierenden Notar bestellt werden.

\section{Abschnitt IV: Zuständigkeiten}

\section{Artikel 10}

Die notariellen Angelegenheiten werden von den Notariaten des Heimatwohnsitzes des Antragstellers, oder des Ortes, wo das Rechtsgeschäft oder die (rechtlich relevante) Tatsache stattgefunden hat, wahrgenommen.

Artikel 11

Für notarielle Angelegenheiten, die die Eigentumsübertragung betreffen, sind die Notariate des Heimatwohnsitzes des Antragstellers oder des Ortes, wo sich das Eigentum in der Hauptsache befindet, zuständig.

Artikel 12

Wenn sich die Heimatwohnorte von mehreren antragstellenden Parteien in Bezug auf ein- und diesselbe notarielle Angelegenheit nicht im Amtsbezirk eines Notariats befinden, oder wenn die Orte, an denen sich das Eigentum befindet, die Amtsbezirke mehrerer Notariate überschneiden, kann nach vorheriger Abstimmung unter den Beteiligten bei einem dieser Notariate ein Antrag gestellt werden. Wenn die Beteiligten keine Einigung erzielen können, wird von den betreffenden Notariaten eine Abstimmung über die Zuständigkeit vorgenommen, so wie es für die Bürger am günstigsten ist.

\section{Artikel 13}

Wenn es zwischen den Notariaten zu Meinungsverschiedenheiten über die Zuständigkeiten kommt, bestimmt die ihnen gemeinsam übergeordnete Justizverwaltungsbehörde die Zuständigkeit.

\section{Artikel 14}

Das Justizministerium sowie die Justizverwaltungsbehörden der Provinzen, Autonomen Gebiete und Regierungsunmittelbaren Städte haben das Recht festzulegen, welche notariellen Aufgaben von welchen Notariaten wahrgenommen werden.

\section{Artikel 15}

Die Botschaften und Konsulate unseres Landes im Ausland können auf Wunsch von im Gastland (residierenden) Bürgern unseres Landes notarielle Angelegenheiten bearbeiten. 


\section{Abschnitt V: Das Verfahren der öffentlichen Beurkundung}

\section{Artikel 16}

Wenn die Beteiligten die öf fentliche Beurkundung beantragen, müssen sie den Antrag persönlich in schriftlicher oder mündlicher Form beim Notariat stellen. Wenn andere Personen beauftragt werden, müssen diese im Besitz eines Dokumentes über ihre Vertretungsmacht sein. Anträge auf Beurkundung und Beglaubigung von Vollmachten, Erklärungen, Adoptionen, Testamenten, Unterschriften und Siegeln dürfen jedoch nicht von anderen Personen vertretungsweise gestellt werden; wenn für die Beteiligten Schwierigkeiten damit verbunden sind, können die Notare diese an ihrem Aufenthaltsort aufsuchen und dort die notariellen Angelegenheiten erledigen.

Staatliche Organe, Verbände, Betriebe und Institutionen müssen, wenn sie Anträge auf öffentliche Beurkundung stellen, ihre Vertreter zum Notariat entsenden. Der Vertreter muß im Besitz eines Dokumentes über seine Vertretungsmacht sein.

\section{Artikel 17}

Die Notare dürfen keine notariellen Angelegenheiten bearbeiten, die sie selbst, ihre Ehepartner sowie ihre nahen Verwandten oder nahe Verwandte der Ehepartner betreffen. Desgleichen dürfen sie keine notariellen Angelegenheiten bearbeiten, die für sie selbst oder für ihre Ehepartner mit Vorteilen oder Nachteilen verbunden sind.

Die Beteiligten haben das Recht des Antrages auf Ablehnung des Notars.

Artikel 18

Die Notare müssen die Identität sowie die Befähigung der Beteiligten, Rechte auszuüben und Pflichten zu übernehmen, nachprüfen. Sie müssen (ebenfalls) nachprüfen, ob die von den Beteiligten zur öf fentlichen Beurkundung beantragten Tatsachen und Urkunden sowie die damit in Zusammenhang stehenden Unterlagen echt und rechtmäßig sind.

Artikel 19

Wenn die Notariate der Ansicht sind, daß die von den Beteiligten beigebrachten Belege unvollständig oder zweifelhaft sind, haben sie das Recht, die Beteiligten zu den notwendigen Ergänzungen aufzufordern oder bei den betreffenden Einheiten und Einzelpersonen nachzuforschen, um relevante Unterlagen und Material zu erhalten. Die betreffenden Einheiten und Einzelpersonen haben die Pflicht, Hilfestellung zu leisten.

Artikel 20

Die Notare müssen entsprechend den vom Justizministerium festgelegten oder genehmigten Formen notarielle Urkunden ausstellen.

Artikel 21

Nach Beendigung der Ausfertigung einer notariellen Urkunde muß ein Duplikat einbehalten werden. Entsprechend dem Bedarf der Beteiligten werden eine Reihe von Kopien angefertigt und diese zusammen mit dem Original den Beteiligten ausgehändigt.

Artikel 22

Die Notariate müssen für die Erledigung der notariellen Angelegenheiten vorgeschriebene Gebühren erheben. Das Verfahren der Erhebung von Notariatsgebühren wird vom Justizministerium gesondert geregelt.

Artikel 23

Das Notariatspersonal muß über die in seinem Notariat bearbeiteten notariellen Angelegenheiten Stillschweigen bewahren.

Artikel 24

Entsprechend den Bestimmungen in Artikel 4, Punkt 10 kann bei Schuldurkunden, auf denen die Vollstreckbarkeit notariell beurkundet wurde, dann, wenn eine der beteiligten Parteien sich nicht an die Bestimmungen der Urkunde hält, die andere beteiligte Partei bei den dafür zuständigen Volksgerichten der unteren Ebene die Vollstreckung beantragen. 


\section{Artikel 25}

Die öffentlichen Notariate müssen die notarielle Beurkundung von Tatsachen und Dokumenten, die nicht der Wahrheit entsprechen und nicht rechtmäßig sind, ablehnen. Wenn die Notariate den Antrag der Beteiligten auf notarielle Beurkundung ablehnen, müssen sie gegenüber diesen mündlich oder schriftlich die Gründe für die Ablehnung erklären, desgleichen müssen sie über das Einspruchsverfahren aufklären, falls die Ablehnung nicht akzeptiert wird.

Wenn die Beteiligten die Ablehnung durch das Notariat nicht akzeptieren oder die Entscheidung des Notariats für unangemessen halten, können sie bei den für den Sitz des Notariats zuständigen Justizverwaltungsbehörden der Kreise und Städte oder bei den höheren Justizverwaltungsbehörden Einspruch erheben, über den von damit befaßten Behörden entschieden wird.

\section{Artikel 26}

Wenn die Notariate oder die für sie zuständigen Justizverwaltungsbehörden oder die höhere Justizverwaltungsbehörden entdecken, daß bereits ausgestellt notarielle Urkunden unangemessen oder fehlerhaft sind, müssen sie diese widerrufen.

Artikel 27

Wenn die Beteiligten notarielle Urkunden beantragen, die für die Verwendung im Ausland vorgesehen sind, müssen diese zusätzlich zu dem Verfahren nach den Bestimmungen in diesem Abschnitt auch an das Außenministerium oder die Büros für Auswärtige Angelegenheiten der Provinzen, Autonomen Gebiete und Regierungsunmittelbaren Städte und an die Botschaft und Konsulate der betreffenden Staaten in China zur Legalisation gesandt werden, es sei denn, der Staat, in dem die Urkunde verwendet werden soll, hat andere Bestimmungen oder es bestehen zweiseitige Abkommen über die Befreiung von konsularischer Legalisation.

\section{Abschnitt VI: Ergänzende Bestimmungen}

Artikel 28

Diese Bestimmungen werden auch auf in China residierende ausländische Bürger angewandt. Artikel 29

Für die Auslegung dieser Bestimmungen ist das Justizministerium verantwortlich.

Artikel 30

Diese Bestimmungen treten am Tage der Verkündung in Kraft. 


\title{
ABSTRACTS
}

\section{Structural Change in International Shipping}

\author{
by Robert Kappel
}

In order to establish a new international economic order the United Nations resolved in 1974 that all efforts should be made to promote an increasing und equitable participation of developing countries in world shipping. Since 1964 UNCTAD and more and more Third World countries increased their efforts to fight against the dominance in international seaborne trade of shipping enterprises of the developed market economies. Especially since the beginning of the seventies as a reaction to the growing flags of convenience (Liberia, Panama), which are mostly property of shipping companies of OECD-countries, the Third World countries' action to participate in international shipping and build up own fleets took place on three main fronts: 1 . The liner sector, where the code of conduct for liner conferences will be put in force in October 1983. 2. The bulk shipping market (cargo sharing in bulk trade). 3. The transformation of flags of convenience. These postulates are the basis for the New International Maritime Order. The aim of the Third World was and is more control over the costs and freight rates and the states' desire to help national shipping companies to transport more national cargo. The article describes the development of shipping activities by UNCT AD and Third World countries, the actual controversies in world shipping, and analyses the state of shipping in the Ivory Coast and South Korea.

\section{Public Notaries in the People's Republic of China}

\section{by Harald Richter}

This article discusses recent developments in a less-known field of the legal system of the People's Republic of China, the public notary offices. On April 13, 1982, the State Council of the People's Republic of China issued the first "Provisional Regulations for the Public Notaries of the People's Republic of China" since the founding of the People's Republic, thus providing a legal basis for the work of public notary offices. Since 1979, public notary offices in various parts of China have resumed activities which had been interrupted for two decades. Following the liberalization of domestic economic life and the rapid development of international relations, there has been an increasing de- 
mand for notary services. The re-establishment of a network of public notary offices is considered part of strengthening the "Socialist Legal System". Public notary offices are state offices, supervised by the departments of justice of the People's Governments at all levels and are responsible for the official authentication and verification of documents and contracts and certain other legal matters. By June 1982, around 1,600 public notary offices had been set up in Chinese counties, districts and cities. This number now exceeds the highest number of such offices existing before the 'Cultural Revolution', around 1,100 in 1957 . So far, the public notary offices have mainly handled international cases, i.e. documents concerning the affairs of non-residents (mostly overseas Chinese) or nonChinese residents. But domestic cases are becoming more and more important.

The article presents a short introduction to the development of the system of public notary offices since 1949, and it further discusses the organization, functions and duties of these of fices, as well as regulations concerning the form of notarial documents. Some statistical data relating to the development between 1979 and 1982 are also given. A German translation of the "Provisional Regulations" is appended.

\section{The Search for National Identity and Language Problems - Somalia's Experience}

\section{by Thomas Labahn}

There are well over 1000 languages in Africa today. Thus African states are faced with the necessity to create official languages which, among other things, promote a sense of overarching nationality, as well as stability and national continuity. A national language is also instrumental to facilitate development. As one of only few African nations Somalia uses its indegenous language as the official language.

The difficulties to use Somali as the official language were compounded by the fact that there existed no script for the orally transmitted language. So, upon gaining independence, English, Italian, and Arabic were used as official languages. This decision overlooked a rising nationalism among Somalia's population and the use of Somali as an official language became a common national goal. As for the script three alternatives have been discussed. Using a modified Arabic as script for The Somali language has always been a favourite solution for the islamic clerics. Nationalists have preferred the creation of a special script for Somali, the Osmania. The military government under Siad Barre, which came to power in 1969 , finally instituted a script using the Latin alphabet, mostly for pragmatic reasons.

The article chronicles historically the discussion preceeding Barre's decision. Correlations between language problems and emerging national identity become visible, too. 